Relations industrielles

Industrial Relations

\title{
Le travail des deux conjoints: Effet sur la progression de carrière du cadre
}

\author{
Jean-Yves Le Louarn, Roland Thériault et Jean-Marie Toulouse
}

Volume 39, numéro 1, 1984

URI : https://id.erudit.org/iderudit/050002ar

DOI : https://doi.org/10.7202/050002ar

Aller au sommaire du numéro

Éditeur(s)

Département des relations industrielles de l'Université Laval

ISSN

0034-379X (imprimé)

1703-8138 (numérique)

Découvrir la revue

Citer cet article

Le Louarn, J.-Y., Thériault, R. \& Toulouse, J.-M. (1984). Le travail des deux conjoints: Effet sur la progression de carrière du cadre. Relations industrielles / Industrial Relations, 39(1), 36-50. https://doi.org/10.7202/050002ar
Résumé de l'article

Les auteurs étudient l'effet du mariage à une personne qui travaille à l'extérieur sur la progression de carrière de l'individu dans l'entreprise.
Tous droits réservés (C) Département des relations industrielles de l'Université Laval, 1984
Ce document est protégé par la loi sur le droit d'auteur. L'utilisation des services d'Érudit (y compris la reproduction) est assujettie à sa politique d'utilisation que vous pouvez consulter en ligne.

https://apropos.erudit.org/fr/usagers/politique-dutilisation/ 


\title{
Le travail des deux conjoints Effet sur la progression de carrière du cadre
}

\author{
Jean-Yves Le Louarn \\ Roland Thériault \\ Jean-Marie Toulouse
}

Les auteurs étudient l'effet du mariage à une personne qui travaille à l'extérieur sur la progression de carrière de l'individu dans l'entreprise.

Le phénomène des couples dont les deux personnes poursuivent une carrière séparée a fait l'objet de plusieurs études au cours des dix dernières années de la part des chercheurs en sciences sociales. Rapoport et Rapoport (1971) sont sans doute les premiers à avoir étudié de façon empirique les problèmes et contraintes auxquels font face ce genre de couples. Presqu'en même temps, Holmstrom (1972) publiait les résultats de sa thèse portant, comme pour les Rapoport, sur un petit échantillon de couples fortement scolarisés. Récemment, St. John Parsons (1978) recommençait l'étude faite par les Rapoport avec un groupe de couples américains. Ces études ont tenté de faire la lumière sur les problèmes auxquels sont confrontés les couples qui choisissent de poursuivre deux carrières.

Il serait certainement utile de faire appel à d'autres perspectives et d'autres méthodes afin de mieux cerner les multiples facettes du phénomène des couples à deux carrières. Entre autres, il semble nécessaire d'étudier ce domaine du point de vue des employeurs concernés. Ce dernier est presque absent de la documentation existante (Rapoport et Rapoport 1978). Il y a cependant quelques exceptions: Rosen et coll. (1975), Berger et Wright (1977) et Le Louarn et DeCotiis (1982).

Les résultats de ces études mettent en lumière les réticences des cadres - américains, au moins - à l'égard des couples à deux carrières. Lorsqu'on leur demande de prendre des décisions telles que embauche, pro-

- LE LOUARN, Jean-Yves, Roland THERIAULT et Jean-Marie TOULOUSE, professeurs, École des Hautes études commerciales, Montréal.

** Les auteurs remercient Lyne Sylvain pour sa précieuse collaboration dans le traitement des données sur ordinateur. 
motion ou mutation, les cadres ont tendance à rejeter plus les membres de couples à deux carrières que les autres. Ceci est surtout vrai lorsqu'il s'agit de muter une personne - même très compétente - d'un endroit à un autre. De telles décisions ont pour effet de freiner la progression de carrière des couples concernés.

Deux études récentes indiquent que les hommes dont la femme travaille à l'extérieur à temps plein gagnent moins que les autres. Ces deux recherches sont intéressantes car elles utilisent deux méthodes assez différentes. Mooney (1981) a utilisé des données recueillies entre 1967 et 1971. Elle n'a retenu que les couples dont les deux personnes avaient été sur le marché du travail à temps plein (plus de 1,200 heures par année) les cinq dernières années. Les résultats montrent que les revenus des maris dont l'épouse ne travaille pas à l'extérieur sont de $20 \%$ supérieurs à ceux des maris dont l'épouse travaille à l'extérieur (le revenu moyen sur cinq ans était de $\$ 21,069$ dans un cas et de $\$ 26,582$ dans l'autre). De plus, le pourcentage monte à $25 \%$ quand le mari a un emploi de bureau $(\$ 24,210$ contre $\$ 31,268)$ plutôt que d'ouvrier $(\$ 17,494$ contre $\$ 20,578)$ ou lorsque l'épouse a plus de douze années de scolarité $(\$ 22,707$ contre $\$ 31,030)$. Bien entendu, ces résultats ne démontrent pas que le fait d'avoir une épouse qui travaille à l'extérieur à temps plein est la cause de la différence de salaire; pas plus que ces résultats n'excluent l'influence d'autres variables sur le salaire annuel. Le problème du contrôle d'autres variables est résolu par les auteurs de la deuxième étude.

Pfeffer et Ross (1982) proposent trois énoncés théoriques, empruntés à divers auteurs, qui prédisent des différences de salaire semblables à celles trouvées par Mooney. (1) Les salaires seraient inférieurs pour les maris dont les femmes travaillent à l'extérieur parce que ces dernières ne se conformeraient pas aux attentes de la société à leur égard. (2) Le mari dont la femme ne travaille pas à l'extérieur peut utiliser les services de cette dernière pour promouvoir sa propre carrière. L'épouse peut, par exemple, aider à développer les relations importantes pour la carrière du mari, conseiller celui-ci au sujet de sa carrière et l'aider à conserver une bonne santé mentale (Kanter, 1977). Un mari qui n'aurait pas accès à ces «services» parce que son épouse travaille à l'extérieur serait désavantagé. Ceci se traduirait dans son salaire. (3) Pfeffer et Ross notent enfin que le fait d'avoir une femme qui travaille à l'extérieur est une indication d'un besoin financier élevé. Dans la mesure où les salaires sont le reflet des besoins financiers des gens, les hommes mariés à une femme qui travaille gagneraient moins que les autres. 
Pour tester ces hypothèses, Pfeffer et Ross (1982) ont utilisé des données recueillies en 1966 auprès d'un échantillon aléatoire américain, composé de 2,595 hommes. Les résultats indiquent que les hommes mariés à une femme qui travaille à l'extérieur avaient, à cette époque, un revenu annuel significativement inférieur aux autres hommes de l'échantillon. Ceci s'avérait vrai tant pour les cadres que pour les professionnels et les «cols bleus», bien que l'effet fût plus prononcé pour les deux premières catégories de répondants. Cet effet négatif demeurait après avoir contrôlé l'effet d'autres variables sur le revenu annuel: la scolarité, la race, l'ancienneté dans l'entreprise, le statut socio-économique, la formation additionnelle reçue, le niveau d'habileté requis par l'emploi, la taille de la ville et le fait d'être marié ou non.

Ces deux études indiquent que les hommes mariés à une femme qui travaille à l'extérieur touchent un salaire inférieur aux hommes mariés à quelqu'un qui ne travaille pas à l'extérieur.

Il convient d'émettre quelques réserves à l'endroit de ces deux études afin d'en montrer les limites. D'abord, les données utilisées par les auteurs ont été recueillies il y a une quinzaine d'années environ. $\mathrm{Si}$, comme le suggèrent Pfeffer et Ross, les différences de salaire sont expliquées par leurs hypothèses 1 et 2 (conformation aux attentes de la société et épouse utilisée comme ressource dans la carrière), il est possible qu'elles se soient estompées à mesure qu'augmentait le nombre de femmes mariées travaillant à l'extérieur. Ensuite, tant les explications théoriques que leur vérification empirique portent sur les différences de salaires chez les hommes seulement. Il est donc impossible de généraliser les résultats aux femmes qui travaillent. La question se pose: dans quelle mesure les propositions théoriques de Pfeffer et Ross (1982), concernant notamment l'utilisation de l'épouse dans la carrière, s'appliquent aussi aux femmes qui font carrière? De plus, ces auteurs ont opérationnalisé le concept de carrière au moyen des variables «salaires» et «prestige occupationnel». Il existe d'autres indices mesurables de ce concept. Le niveau hiérarchique atteint par la personne et le nombre de promotions obtenues en sont deux exemples.

\section{HYPOTHĖSES}

Le but de cette recherche est d'étudier l'effet du mariage à une personne qui travaille à l'extérieur sur la progression de carrière de l'individu dans l'entreprise. En ce sens, notre étude poursuit les travaux entrepris par Mooney (1981) et Pfeffer et Ross (1982). Le concept de progression de carrière est ici mesuré par trois variables: (1) le niveau de salaire, (2) le niveau 
hiérarchique et (3) le nombre de promotions obtenues par l'individu. Le fait de mesurer la variable dépendante de plusieurs façons ne peut qu'augmenter la validité des résultats obtenus.

En ce qui a trait à la variable indépendante, il apparaît souhaitable de dissocier conceptuellement un(e) conjoint(e) qui travaille à temps partiel et à temps plein. Si l'on retient la théorie de Kanter (1977) sur l'utilisation du conjoint comme ressource, il est certain qu'un conjoint qui travaille à temps partiel peut continuer à jouer en partie son rôle de ressource auprès de l'individu. L'effet sur la progression de carrière devrait être plus sensible, plus le conjoint travaille à l'extérieur.

De plus, il a été signalé plus haut que la théorie de Kanter (1977), sur laquelle s'appuient Pfeffer et Ross (1982), reposait sur l'homme au travail, la femme à la maison jouant le rôle de support à la carrière de son mari. Il apparaît important de vérifier si cet énoncé théorique demeure valable dans la situation inverse. Le fait d'avoir un conjoint qui poursuit une carrière a-t-il aussi un effet négatif sur la progression de carrière des femmes?

Enfin, comme l'ont noté Anderson et coll. (1981), les caractéristiques de l'entreprise peuvent affecter les critères de mobilité interne. D'autre part, Margolis (1979) a observé que, contrairement aux organisations gouvernementales, les règles d'avancement dans les grandes entreprises privées étaient loin d'être claires et les promotions ressemblaient beaucoup à des nominations politiques. Enfin, Lawler (1966) a montré que les critères de détermination des salaires étaient différents dans le secteur public et dans le secteur privé. Il a notamment observé que l'ancienneté était reliée positivement au salaire dans le premier cas et négativement dans le second, que la qualité de la performance au travail avait un lien avec le salaire seulement dans le privé et qu'il en était de même pour l'effort déployé par l'individu. Ces différentes observations nous amènent à penser que la carrière d'un individu ne progresse pas selon les mêmes règles dans les secteurs privés et publics. Il apparaît donc intéressant de vérifier si l'effet de notre variable indépendante sur les trois variables dépendantes est le même pour les deux secteurs.

En terminant, nous retenons dans cette étude l'idée de Pfeffer et Ross (1982) que nos trois indices de progression de carrière sont influencés par d'autres variables que le statut du conjoint. Il est donc nécessaire d'effectuer les contrôles appropriés.

Les hypothèses proposées sont donc les suivantes: (1) plus le conjoint travaille en dehors du foyer, moins le salaire de l'individu, son niveau hiérarchique et le nombre de promotions qu'il a obtenues sont élevés, tout en 
contrôlant l'effet des variables suivantes: l'âge, la scolarité, la langue, le sexe, le statut civil, le secteur d'activités, le nombre total d'employeurs pour lesquels la personne a travaillé, l'ancienneté et la performance. Pour la variable «salaire», les variables «niveau hiérarchique» et «nombre de promotions» font également partie des variables de contrôle. (2) L'effet du travail du conjoint à l'extérieur du foyer sur le salaire, le niveau hiérarchique et le nombre de promotions de l'individu est différent selon que ce dernier oeuvre dans le secteur privé ou dans le secteur public. Il en est de même selon que l'individu est un homme ou une femme.

\section{MÉTHODE}

\section{Population et méthode de cueillette de l'information}

Les données utilisées dans ce rapport proviennent d'un projet de recherche beaucoup plus vaste portant sur les cadres et leur travail dans les organisations au Québec'.

La population étudiée est constituée de cadres des différentes unités administratives à l'intérieur des organisations et oeuvrant à divers paliers hiérarchiques (contremaître ou superviseur jusqu'à vice-président ou président). Également, vu la dualité linguistique et culturelle du monde du travail québécois, tant les cadres francophones qu'anglophones font partie de la population utilisée. Dans cette étude, la taille de l'échantillon est de $\mathbf{1 7 5 0 .}$

\section{Instruments de mesure}

Le degré de progression dans la carrière fut opérationnalisé de trois façons distinctes: (1) le niveau de salaire annuel de la personne $(1=$ inférieur à $\$ 15,000,11=\$ 60,000$ et plus); (2) le niveau hiérarchique de la personne $(1=$ le niveau de la haute direction, soit le président, le directeur général et ses quelques collaborateurs immédiats formant l'équipe de direction, 7 = le plus bas niveau de supervision, soit les contremaîtres et le personnel de supervision le plus proche des exécutants); (3) le nombre total de promotions de la personne depuis qu'elle travaille à temps plein.

Quant à la variable indépendante, soit celle servant à identifier si le conjoint du répondant travaillait à l'extérieur du foyer, elle fut mesurée au moyen d'une variable créée à partir de deux questions recodifiées et pouvant

1 Les professeurs Roland Thériault et Jean-Marie Toulouse, de l'École des Hautes Études commerciales de Montréal, sont les responsables de ce projet de recherche portant sur les cadres et leur travail dans les organisations au Québec. 
prendre les valeurs suivantes: 0 , si le conjoint ne travaillait pas à l'extérieur; 1 , s'il travaillait à l'extérieur à temps partiel ou de façon temporaire; 2 , s'il travaillait à l'extérieur à temps plein et durant toute l'année.

Finalement, en ce qui a trait aux variables servant d'éléments de contrôle, les variables âge, sexe, nombre d'années d'expérience de travail à temps plein (ancienneté totale) et le nombre total d'employeurs pour lesquels la personne a travaillé furent mesurées directement. Le degré de scolarité fut mesuré en demandant au cadre d'indiquer à quel niveau il en était lorsqu'il a abandonné ses études: études primaires (1), secondaires, etc. jusqu'à doctorat (7). Le degré de performance de la personne était mesuré en lui demandant d'indiquer sur une échelle de quatre points l'opinion de son supérieur immédiat concernant sa performance au travail (1 signifie rendement exceptionnel et 4 , rendement inférieur aux autres). La variable «langue» fut mesurée en demandant au répondant d'indiquer s'il se considérait francophone ou anglophone. Quant à la variable «statut civil, bien qu'il y eût plusieurs réponses possibles, elle fut recodifiée de façon à séparer les personnes mariées de toutes celles qui vivaient seules (célibataire, veuf(ve), etc.).

\section{RÉSULTATS}

Les tableaux 1A et 1B présentent la description des diverses variable utilisées.

TABLEAU 1A

Moyennes et écarts-types des variables continues

$\begin{array}{lrc}\text { Variables } & \text { Moyenne } & \text { Écart type } \\ \text { Niveau hiérarchique } & & \\ \quad(1=\text { haut; } 7 \text { = bas }) & 4,02 & 2,08 \\ \text { Nombre de promotions } & 3,02 & 2,37 \\ \text { Âge } & 41,37 & 8,88 \\ \text { Nombre total d'employeurs } & 3,14 & 1,96 \\ \text { Ancienneté totale } & 19,62 & 9,14 \\ \text { Performance } & & \\ (1=\text { exceptionnelle; } 4=\text { inférieure }) & 2,41 & 0,64\end{array}$


TABLEAU 1B

Distribution des variables discontinues

$\begin{array}{llc}\text { Variables } & \text { Catégories de réponses } & \text { Pourcentages } \\ \text { Salaires } & - \text { moins de } \$ 24,999 & 12 \\ & -\$ 25,000-\$ 34999 & 30 \\ & -\$ 35000-\$ 44999 & 31 \\ \text { Conjoint travaille } & -\$ 45000 \text { et plus } & 27 \\ & - \text { pas } & 49 \\ & - \text { à temps partiel } & 18 \\ \text { Scolarité } & - \text { à temps plein } & 33 \\ & - \text { secondaire ou moins } & 18 \\ & - \text { post-secondaire et collégial } & 22 \\ \text { Langue } & - \text { baccalauréat } & 33 \\ & - \text { maîtrise ou plus } & 27 \\ \text { Sexe } & - \text { francophones } & 93 \\ & - \text { anglophones } & 07 \\ \text { Statut civil } & - \text { hommes } & 80 \\ & - \text { femmes } & 20 \\ \text { Secteur d'activités } & - \text { vivent seuls } & 15 \\ & - \text { mariés } & 85 \\ & - \text { public } & 78 \\ & & 22\end{array}$

Pour vérifier nos hypothèses, nous avons effectué deux analyses. D'abord, nous avons calculé des corrélations entre la variable indépendante et les trois variables dépendantes. Ensuite, nous avons procédé à des régressions multiples afin de tenir constant l'effet des variables de contrôle.

La première analyse montre bien des relations inverses entre le salaire $(-0.16)$, le niveau hiérarchique $(0,09)$, le nombre de promotions obtenues $(-0,10)$ et l'implication du conjoint dans un emploi à l'extérieur du foyer. Ces relations sont modestes mais toutes significatives au seuil de 0,01 . Quelques autres corrélations significatives au même seuil méritent d'être signalées; celle entre l'implication du conjoint dans un travail à l'extérieur du foyer et l'âge du cadre $(-0,20)$, les années d'expérience de travail $(-0,22)$ et le niveau d'instruction $(0,05)$. Enfin, soulignons que les cadres du secteur public se situent à un niveau hiérarchique supérieur à ceux du secteur privé $(0,27)$. Il se peut que, dans le secteur privé, il y ait moins d'unités économiques représentées ou bien que la structure organisationnelle du secteur privé soit différente de celle du «public», ou encore que, compte tenu de la 
mesure subjective du niveau hiérarchique, les cadres du secteur public aient plus tendance à se situer à des niveaux supérieurs. Cette relation entre le secteur d'activités et le niveau hiérarchique ne peut être éclaircie par nos données. Bien qu'il n'y ait pas de relation conceptuelle entre les deux variables mais compte tenu de la corrélation constatée, nous avons décidé de contrôler l'effet de la variable "secteur d'activités» dans les analyses ultérieures.

Les résultats des régressions se retrouvent aux tableaux 2 et 3 .

L'hypothèse sur la relation entre le salaire du cadre et le fait que son conjoint travaille à l'extérieur est confirmée. Plus le conjoint est engagé dans un travail à temps plein à l'extérieur du foyer, plus le niveau de salaire du cadre est bas. Toutefois, les hypothèses ne sont pas confirmées quant aux relations avec le niveau hiérarchique et le nombre de promotions lorsque l'on contrôle pour un certain nombre de caractéristiques conceptuellement reliées à ces variables. Cependant, tant dans le cas de la variable niveau hiérarchique que dans celui du nombre de promotions, les variables secteurs d'activités et sexe ont des effets significatifs. Il est alors possible que les effets des variables sexe et secteur d'activités sur le niveau hiérarchique et le nombre de promotions soient contraires d'un sexe à l'autre et d'un secteur d'activités à l'autre et, ainsi, ces effets s'annulent lorsque l'on ne fait pas de distinctions à l'intérieur de chacune de ces variables (hypothèse 2).

Le tableau 3 présente l'ensemble des régressions reliées à la distinction «privé-public». Comme on peut le constater, l'hypothèse 2 est confirmée uniquement pour la variable salaire. En effet, même en contrôlant pour un ensemble de variables pertinentes, le salaire du cadre dont le conjoint travaille à l'extérieur est moindre pour la personne qui oeuvre dans le secteur public. Cette relation n'est pas confirmée pour les cadres qui oeuvrent dans le secteur privé.

Finalement, ce même tableau 3 présente les régressions pertinentes selon que le cadre est un homme ou une femme (deuxième partie de l'hypothèse 2). À nouveau, l'hypothèse concernant le salaire est confirmée alors que celles portant sur le niveau hiérarchique et le nombre de promotions ne le sont pas lorsque l'on contrôle pour un ensemble de variables pertinentes. Quant au salaire, il est inférieur pour les cadres de sexe masculin dont le conjoint travaille à l'extérieur alors que cela n'est pas le cas pour les cadres de sexe féminin. 


\section{TABLEAU 2}

Importance du fait que le conjoint travaille sur la progression de carrière du cadre

Indices de progression de carrière

Variables explicatives

\begin{tabular}{|c|c|c|}
\hline $\begin{array}{c}\text { Salaire } \\
(N=2003)\end{array}$ & $\begin{array}{c}\text { Niveau } \\
\text { hiérarchique } \\
(N=2016)\end{array}$ & $\begin{array}{c}\text { Nombre de } \\
\text { promotions } \\
(N=2030)\end{array}$ \\
\hline$F$ & $B$ & $\boldsymbol{B}$ \\
\hline
\end{tabular}

Conjoint travaille

( $1=$ non; $3=$ temps plein)

$-, 13$

$14,13^{* *} \quad, 05$

0,84

, 04

0,46

Âge

, 03

$14,21 * * \quad, 03$

$7,56^{* *}$

$-, 03$

$0,72^{*}$

Scolarité

, 56

$480,38^{* *}-, 30$

64,04

, 16

$13,63^{* *}$

Langue

$(1=$ franco $; 2=$ anglo $)$

$-, 30$

$7,32^{* *}-, 05$

0,10

$, 25 \quad 1,76$

Sexe

(1 = homme; 2 =femme)

$-, 85$

Statut civil

( 1 = autres; 2 =marié)

Secteur d'activités

( $1=$ public; 2 = privé)

$\begin{array}{llllll}, 24 & 6,75^{* *} & -, 19 & 2,09 & , 03 & 0,03\end{array}$

Niveau hiérarchique

( 1 = haut; 7 = bas)

$-, 69$

,69 $67,72^{* *} \quad, 84 \quad 48,50^{* *} \quad, 43 \quad 9,12^{* *}$

- ,28 346,39** - -

Nombre de promotions

, 15

$125,18^{* *}$

$\begin{array}{lll}-, 04 & 5,63^{*} \quad, 02\end{array}$

1,70

,06

$7,68^{* *}$

,01 3,08

, 00

0,15

, 12

$63,46^{* *}$

Performance

(1 = exceptionnelle;

4 =inférieure)

$-, 10$

$4,60^{*}$

,39

$36,58^{* *}$

$-, 38$

$24,38^{* *}$

Constante

4,86

5,05

2,47

$\mathrm{R}^{2}$ ajusté

, 63

$287,30^{* *} \quad, 24$

$60,18^{* *} \quad, 16$

$39,44^{* *}$ 


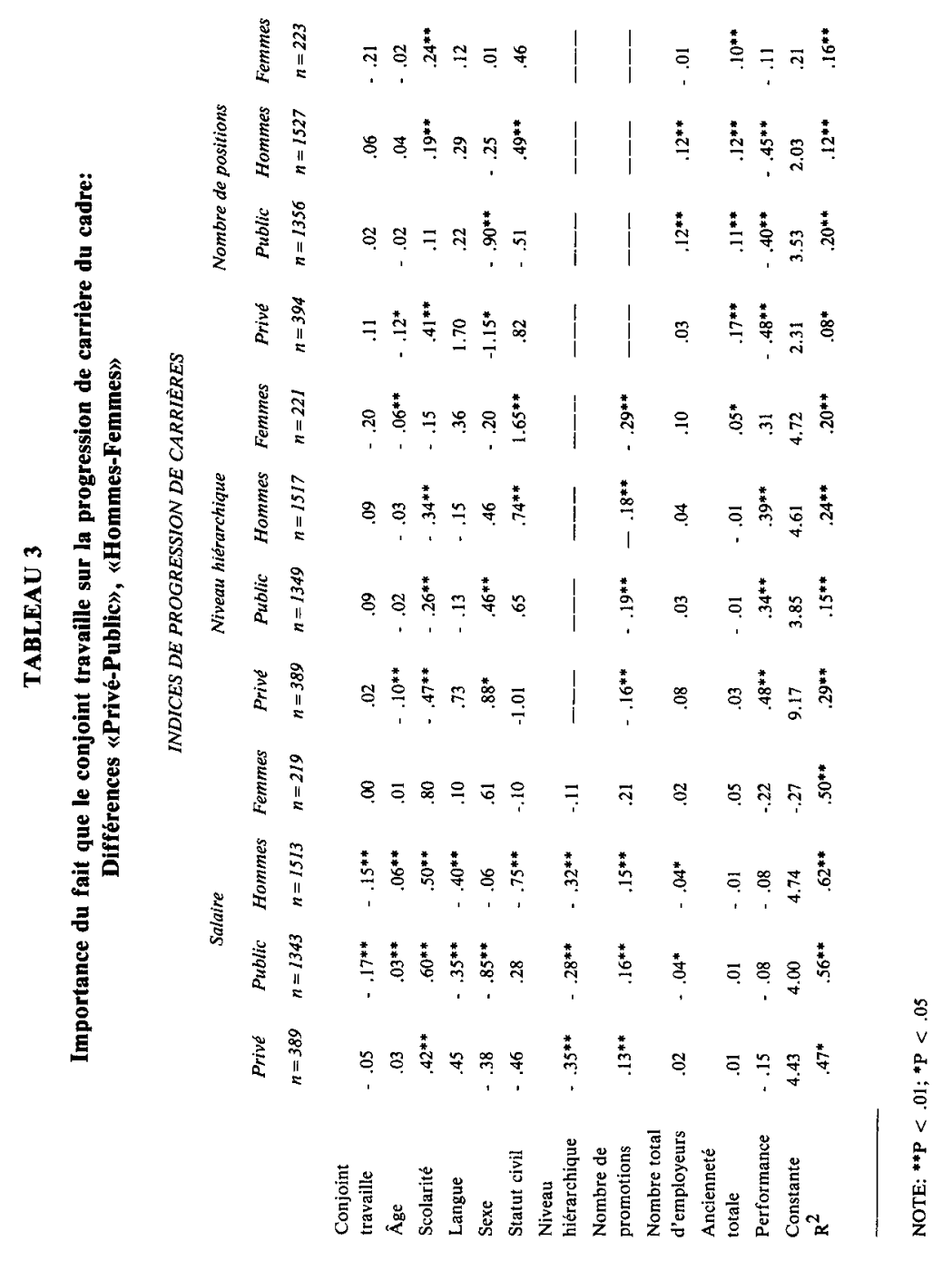




\section{DISCUSSION}

Cette recherche s'inscrit dans le cadre de l'étude de l'impact des couples à deux carrières sur la vie organisationnelle. Elle visait à examiner la relation entre ce phénomène et certains indices de progression de carrière dans les entreprises. Les résultats indiquent que le fait d'avoir un conjoint qui travaille à l'extérieur est associé à un salaire inférieur, si la personne travaille dans le secteur public et si le cadre est de sexe masculin, même après avoir contrôlé l'effet d'un certain nombre de variables. Ces résultats appellent des commentaires et suggèrent plusieurs avenues de recherche.

Premièrement, les résultats concernant le salaire confirment ceux obtenus par Mooney (1981) et Pfeffer et Ross (1982) auprès d'échantillons différents. Bien sûr, le pourcentage de variance expliqué par la variable indépendante n'est pas très élevé. Le fait que le conjoint travaille n'est pas, bien entendu, la variable la plus importante qui agit sur le salaire d'un cadre. L'effet est cependant significatif et peut avoir une signification sociale $^{2}$. Les résultats de notre étude suggèrent un lien (négatif) entre le fait pour un couple de travailler et le niveau de salaire de celui-ci. Si cela s'avérait être une «sanction» du milieu organisationnel - ce qui n'est pas certain — à l'encontre des couples qui travaillent, il y aurait là matière à réflexion. De toute façon, la question mérite d'être étudiée de nouveau.

Deuxièmement, le fait que les deux autres indices de carrière ne soient pas affectés par la variable indépendante peut s'expliquer de plusieurs façons. (1) Le phénomène des doubles carrières est trop récent pour que l'effet ait eu le temps de se faire sentir sur ces deux mesures de la progression de carrière du cadre au Québec. (2) L'effet n'apparaît pas parce que nous n'avons pas contrôlé depuis combien de temps le conjoint était sur le marché du travail. Il faut peut-être que le conjoint ait travaillé longtemps pour que cela se reflète sur ces deux indices de carrière. (3) L'effet sur ces deux variables ne se fait sentir que si le conjoint a des motivations autres que financières pour poursuivre une carrière. Dans la mesure où la personne veut à tout prix grimper dans la hiérarchie organisationnelle, le fait que son conjoint poursuive une carrière n'aurait aucun effet. (4) L'ascension du cadre ne serait ralentie que lorsque son conjoint a une carrière au sens strict du terme. Le cadre, par exemple, serait «contraint» de refuser une mutation intéressante afin de ne pas être séparé du conjoint qui ne tient pas à abandonner sa carrière. Ceci ne serait pas le cas si le conjoint a un emploi qu'il

2 Rappelons à ce sujet que la corrélation entre le fait de fumer ou non et le cancer du poumon n'est que de 0,07 , ce qui n'a pas empêché certains gouvernements de prendre des mesures en la matière. 
peut abandonner sans problèmes. Il se peut que, dans ce cas seulement, certaines décisions de carrière (promotion, transferts, etc.,...) du conjoint affectent celles du cadre.

Troisièmement, le fait que les hypothèses ne soient pas confirmées dans le secteur privé peut s'expliquer aussi. (1) Nous faisons face à deux groupes de cadres différents. Par exemple, ceux du secteur privé sont nettement moins scolarisés $(\overline{\mathrm{X}}=2,96)$ que ceux du secteur public $(4,86)$. Peut-être aussi que ceux qui entrent dans le secteur public sont moins «agressifs» face à leur carrière et plus préoccupés par leur vie familiale. Il s'en suivrait qu'ils seraient prêts à faire des compromis au détriment de leur progression salariale. (2) Dans le secteur privé où l'accent est sans doute plus mis sur la compétition entre cadres, il n'y a pas autant de place pour des compromis famille-carrière. Il faut progresser, carrière du conjoint ou non, ou bien s'en aller. D'où l'absence d'effet dans le secteur privé.

Quatrièmement, le fait que le niveau salarial soit seulement affecté dans le cas des hommes nous amène aussi à spéculer sur ce résultat. (1) Il se peut que les hypothèses formulées par Kanter (1977) soient surtout valables pour les hommes. Nos résultats semblent aller dans ce sens. (2) Comme dans le cas des secteurs d'activités, le groupe de femmes est différent de celui des hommes rendant les comparaisons impossibles. Nous ne savons rien des traits de personnalité des deux groupes mais nous remarquons que les femmes cadres sont en moyenne plus jeunes ( 39 ans contre 42,1 ans), ont moins d'expérience de travail $(15,95$ ans contre 20,46$)$, se situent à un niveau hiérarchique inférieur $(4,72$ contre 3,84$)$ et ont obtenu moins de promotions $(3,82$ contre 4,65$)$.

Cinquièmement, en ce qui a trait aux hypothèses non confirmées par nos résultats, il faut aussi noter la possibilité pour ces derniers d'être «bons», les hypothèses étant, elles, «mauvaises». Peut-être n'avons-nous pas trouvé d'effet parce qu'il n'y en a tout simplement pas. Il se peut, par exemple, que les effets que nous pourrions observer soient absents parce que le couple s'arrange pour qu'il n'y en ait pas. C'est la vie familiale qui écope et non la progression de carrière! Ensuite, il convient aussi de préciser que l'effet négatif sur la progression de carrière n'est pas le fait de l'organisation qui "sanctionnerait» les couples à deux carrières, bien que des études antérieures semblent l'indiquer (Rosen et coll., 1975; Berger et Wright, 1977; Le Louarn et DeCotiis, 1982), mais plutôt celui des membres du couple qui refusent les règles du jeu organisationnel et «se sanctionnent» euxmêmes. 
Enfin, à la suite de cette étude, il y a lieu de se demander si le fait d'être membre d'un couple à deux carrières a un impact sur d'autres aspects de la vie organisationnelle? Par exemple, les réactions du cadre face à son travail, son niveau de satisfaction (Fukami et coll., 1980) ou son désir d'implication dans l'organisation.

\section{RÉFÉRENCES}

ANDERSON, J.C., G.T. MILKOVICH et A. TSUI, «A Model of IntraOrganizational Mobility», Academy of Management Review, 6, Octobre 1981, 529-538.

BERGER, M. et L. WRIGHT, The Employment of Dual Career Couples, Washington, D.C., U.S. Department of Commerce, N.T.I.S., 1977.

BLAU, P. et O. DUNCAN, The American Occupational Structure, New York, Wiley, 1967.

FUKAMI, C.U., D.Y. FOX et R.J. DUNNE, «The Effect of Dual Career Marriages on Work Related Outcomes", Working Papers Series no. 475, S.U.N.Y. at Buffalo, 1980.

HALL, D.T., Careers in Organizations, Santa Monica, Goodyear, 1976.

HOLMSTROM, L.L., The Two-Career Family, Cambridge, Schenkman, 1972.

KANTER, R.M., Men and Women of the Corporation, New York, Basic Books, 1977.

LAWLER, E.E. III, «Managers' Attitudes Toward How their Pay is and Should be Determined», Journal of Applied Psychology, 50, août 1966, 273-279.

LE LOUARN, J.Y. et T.A. DECOTIIS, «The Effect of Working Couple Status on the Decision to Offer Geographic Transfer», Paper presented at the Annual Meeting of the Academy of Management, New York, 1982.

MARCH, J.C. et J.G. MARCH, «Performance Sampling in Social Matches», $A d$ ministrative Science Quarterly, 23, septembre 1978, 434-453.

MARGOLIS, D.R., The Managers, New York, Morrow, 1979.

MOONEY, M., «Does it Matter if his Wife Works?», Personnel Administrator, 26, janvier 1981, 43-49.

PFEFFER, F.R. et J. ROSS, «The Effects of Marriage and a Working Wife on Occupational and Wage Attainment», Administrative Science Quarterly, 27, mars $1982,66-80$. 
RAPOPORT, R. et R.N. RAPOPORT, Dual-Career Families, Baltimore, Penguin, 1971.

-___, (édit.), Working Couples, New York, Harper and Row, 1978.

ROSEN, B., T.H. JERDEE et T.L. PRESTWICH, «Dual-career Marital Adjustment: Potential Effects of Discriminatory Managerial Attitudes», Journal of Marriages and the Family, 37, août 1975, 565-572.

SCHEIN, E.H., Career Dynamics, Readings, Addison-Wesley, 1978.

ST. JOHN PARSONS, D., Career and Family: A Study of Continuous Dual-Career Families, Thèse de doctorat non publiée, Columbia University, 1978.

\section{Working Spouses' Impact on Managers' Career Progression}

More and more couples are working full-time in Canada giving rise to the dualcareer phenomenon. On the other hand, more and more social scientists are doing research on careers from different points of view including the one adopted in this study; that is, the organizational point of view.

Since working couples, and especially dual-career couples, are more numerous, it seems appropriate to study their potential effects on organizational life. We know almost nothing about the impact on an organization of having members of dualcareer couples, in terms of performance, job satisfaction, absenteism, turnover, etc. Rapoport and Rapoport (1978) noted the absence of the employer's point of view in research on working couples. There are a few exceptions.

Rosen et al (1975), Berger and Wright (1977) and Le Louarn and DeCotiis (1982) have studied the effect of a dual-career status on several personnel decisions within organizations. The results of the empirical studies suggest that being a member of a dual-career couple could be a hindrance when it comes to making important decisions like hiring, promoting, relocating, etc. In those cases, employers belonging to a dual-career couple are perceived as a greater risk for an organization than a member of a single career family. It is feared that if the spouse does not like the new place, or finds another job somewhere else, the couple (including the newly hired or transferred employee) will leave the organization (Holmstrom, 1972).

Following these three studies, one can ask whether some of these decisions have an impact on the career progression of the manager when he or she is a member of a working couple. Mooney (1981) and Pfeffer and Ross (1982) have already shown the negative effect on salary of being married to somebody who works. The purpose of this study is to pursue this line of research by studying the impact of a working couple status on managers' career progression using three different definitions of this construct. 
It is hypothesized that: (1) the more a spouse works outside home, the less is the manager's salary, hierarchical level and number of promotions obtained when the effect of certain variables is controlled; (2) the effect of the working spouse on these three variables will be different according to the manager's sex and whether he or she works in the public or private sector.

Data were collected by means of a questionnaire sent to managers from Québec as part of a larger study conducted by the last two authors. The data from the public sector come from all segments of the governmental bureaucracy. Those from the private sector come from a chain of retail food stores. Data were collected at two different periods of time in 1982 and represent all the hierarchical levels of the organization. The total sample size is 2324 (1817 from the public and 507 from the private sector).

The results show that the salary level of the respondents is negatively related to the working spouse status. That is, when the effects of age, education, spoken language (English or French), sex, civil status (married or not), sector (public or private), hierarchical level, number of promotions, number of employers, length of service and global performance are controlled, the manager who is married to a working spouse has significantly lower salary that the one who is married to somebody who does not work outside the house. Having a working spouse, however, has no significant effect on the manager's hierarchical level on his or her number of promotions. When the sample is divided into two parts, the results of the multiple regression shows that the negative effect on salary is only true in the public sector and for the men only.

Different explanations for these results results are presented and suggestions are made for future research on the effect of dual-career couples on organizational life.

\section{LA SYNDICALISATION DANS LE SECTEUR PRIVÉ AU QUÉBEC}

Préface: Gilles FERLAND - Introduction: Jacques BELANGER, Jean BOIVIN, Claude RONDEAU, Jean SEXTON - Le syndicalisme dans l'entreprise: tendances récentes et analyse, Claude RONDEAU et Jacques BELANGER - La syndicalisation dans le contexte économique québécois, Sidney INGERMAN - Commentaires: Bernard BONIN - L'impact du régime de relations du travail sur la syndicalisation, Gilles LAFLAMME - Commentaires: Hélène LEBEL - Les idées de réforme sur la syndicalisation au Québec depuis 1964, Jacques DESMARAIS - Table ronde: Organisation syndicale: difficultés et motifs de résistance, Pierre MERCILLE, Bertin NADEAU, Madeleine OLIVIER - Pourquoi et comment accroître la syndicalisation dans le secteur privé? Monique SIMARD, Raymond SLIGER - Commentaires: Gérard DION -L'entreprise peut-elle se passer d'un syndicat?, Charles PERREAULT - Commentaires: Fernand GAUTHIER, Paul-Martel ROY - La politique gouvernementale en matière de syndicalisation, Raynald FRÉCHETTE - Table ronde: Les réactions du milieu, Claire BONENFANT, Ghislain DUFOUR, Jean-Paul HÉTU - Syndicalisme, nouvelles technologies et incertitudes économiques, Guy CAIRE.

ISBN 2-7637-7030-4

1 volume, 276 pages - Prix $\$ 17.00$

Les Presses de l'Université Laval

Cité universitaire

C.P.2447, Québec, P.Q., Canada, GIK 7R4 